\title{
Cleidocranial Dysplasia in Saudi Child; A Case Report
}

\author{
Abdullah Almutairi ${ }^{1}$, Ahmad Moosa Alhazmi ${ }^{2}$, Jawaher Jazaa Alotaibi ${ }^{3}$, Abeer AlKhaldi ${ }^{4}$, Ahmed AlQatie ${ }^{5}$, Badi
} ALEnazi $^{6 *}$

\footnotetext{
${ }^{1,2}$ Pediatric Resident at Pediatric Department, Alyamamh Hospital, Riyadh, Saudi Arabia

${ }^{3}$ Medical Student at Collage of Medicine, Princess Nourah Bint Abdulrahman University, Riyadh, KSA

${ }^{4}$ Pharmacist at Ministry of Health

${ }^{5}$ Consultant Radiologist, Department of Radiology, King Faisal Specialist Hospital \& Research Center, Riyadh, KSA

${ }^{6}$ Consultant Pediatric Endocrinologist, Pediatric Department, Alyamammh Hospital, Riyadh, KSA
}

Cleidocranial dysplasia is a rare inherited disease that is characterized by abnormalities in bone such as skull, jaws and shoulder girdle. The present case is a boy who was presented with previous history of fever and NICU admission for 1 week for pneumonia. The boy's parents were first degree consanguinity with no history of similar condition or chronic disease, there were no problems during pregnancy or delivery. The patient showed normal clinical examination and laboratory investigations, whereas radiological examination showed abnormalities in chest, skull and lateral neck. The family of the patient had no history of such condition, the boy suffered this rare disease and this wasn't inherited as this disease is dominant trait, so the mutation caused the disease in our patient is a sporadic.

Keywords: Cleidocranial Dysplasia, Children, RUNX2.

Copyright @ 2020: This is an open-access article distributed under the terms of the Creative Commons Attribution license which permits unrestricted use, distribution, and reproduction in any medium for non-commercial use (NonCommercial, or CC-BY-NC) provided the original author and source are credited.

\section{INTRODUCTION}

Cleidocranial dysplasia (CCD) was derived from an ancint Greek word cleido which means collar bone and kranion which means head and dysplasia which means abnormal formation. It is also known as Scheuthauer-Marie-Sainton syndrome or cleidocranial dysostosis. CCD is an inherited rare skeletal disorder which is characterized by abnormal dental and skeletal development. CCD prevalence is estimated to be one per million with no variation in prevalence regarding race or gender [1]. CCD is usually encountered first by dentists during the routine oral examination [2]. CCD is caused by the mutation of the RUNX2 gene which is located on the short arm of chromosome $6[3,4]$. RUNX2 gene codes for a protein called core-binding transcription factor alpha 1 (CBFA1) which intricate in the bone construction and osteoblasts differentiation [5]. There are 62 types of mutations have been identified in CBFA1/RUNX2 among CCD patients including insertion, deletion, miss sense and non-sense mutations and change in splicing site [6-8]. Here, we present a case of CCD.

\section{Case Presentation}

The present case is a 33 months (almost 3 years) old boy that was presented with history of fever, cough and shortness of breathing for 3 days as well as vomiting and diarrhea for 1 day. The case was Full-term boy with no complication during pregnancy and delivered vaginally with birth weight of $3.4 \mathrm{~kg}$, with history of NICU admission for one week for pneumonia. He had two previous hospitalizations because of pneumonia. His development is appropriate with his age and he is sharing normal family diet. Regarding immunization history, he missed 18 month vaccination. His parents with first degree of consanguinity, father is 30 years old, healthy, employee and mother is 24 years old, healthy, house wife. No family history of similar condition, no family history of chronic disease, there is any current medication or known allergies, they live in Riyadh with average socioeconomic status.

Clinical examination: On examination, child looks unwell, in respiratory distressed, not dehydrated not pale, not jaundiced and no dysmorphic features.

Vital signs included; Temperature $37 \mathrm{C}^{\circ}$, Heart Rate 122 beat/minute Blood Pressure 110/68 mmHg, Respiratory Rate: 50 breath/minute, Oxygen Saturation 95\% on Room air. Growth Parameter: Weight 10 Kilogram (on 3rd percentile) Height 78 centimeter 
(below 3rd percentile) Head Circumference: 51 centimeter on 90th percentile).

Head, Ear, Eye, Nose were Normal, Throat was congested. He had supernumerary teeth, prominent forehead, cardiovascular system examination revealed normal, audible first and second heart sounds, no murmur. Capillary Refill Time: less than 2 seconds. Respiratory system examination showed Clear, Good air entry, no added sound with deformity in the appearance of the chest (pectus excvantum). Abdomen Central Nervous System and Skin all were Normal.

\section{Laboratory investigations}

Investigations including Cell Blood Count (CBC), kidney functions, liver functions and microbiology culture. $\mathrm{CBC}$; White Blood Cell $=5.3$ Red Blood Cell $=5$ Hemoglobin $=12.5$ Mean Corpuscular Volume $=72$ Mean Corpuscular hemoglobin $=24.59$ Mean corpuscular hemoglobin concentration $=33.8$ Platelets $=274 \quad$ NEUT $\%=74$ LYMP $\%=17$ NEUT $=3.9$ LYMPH $=0.9$ Kidney Function Test: Glucose $=7.7$ mmole Blood Urea Nitrogen $=3.5$ mmole Creatinine $=41$ umole Uric Acid $=154$ umole Sodium $=144$ mmole Potassium $=4.1$ mmole Chloride $=104$ mmole Calcium $=2.2$ mmole Liver Function test: Aspartate Transaminase $=61 \mathrm{U} / \mathrm{L}$ Alanine Transaminase $=35 \mathrm{U} / \mathrm{L}$ Alkaline Phosphatase $=144 \mathrm{U} / \mathrm{L}$ Total Bilirubin $=73$ umole Albumin $=41$ Protein $=73$ g/DL. Microbiology Results: Blood culture: No growth.

\section{Radiography}

Radiograph of the skull showing widened sutures and anterior fontanel with multiple wormian bones, mild midface (maxilla) hypoplasia (figure 1).

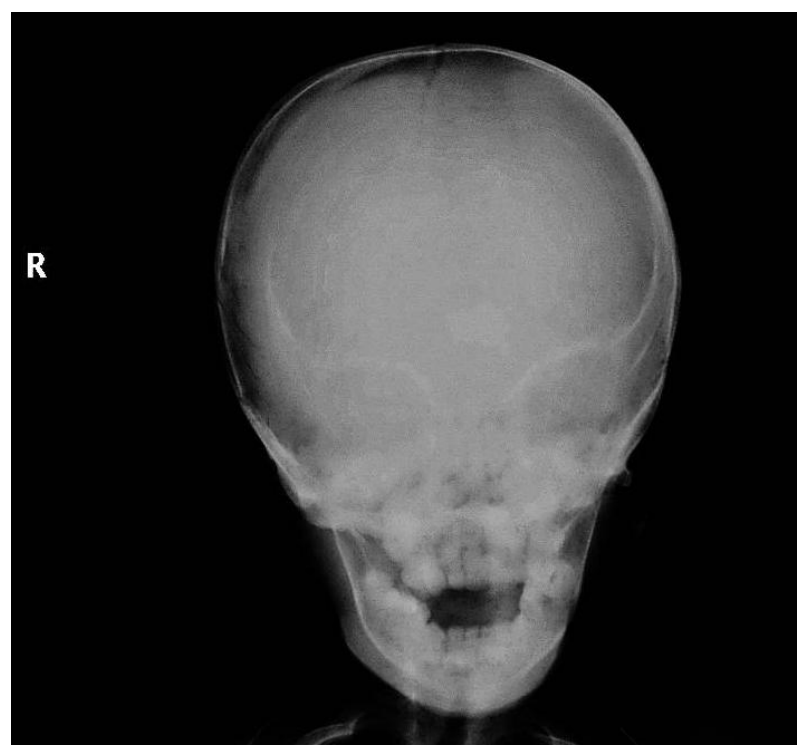

Fig-1: AP radiograph of the skull showing widened sutures and anterior fontanel with multiple wormian bones, mild midface (maxilla) hypoplasia
Chest radiograph showing severely hypoplastic lateral clavicles, slightly small and high scapulae, 11 pairs of ribs and mild levoscoliosis of the lower thoracic and upper lumbar spine. There is also airspace disease in the left lung (figure 2).

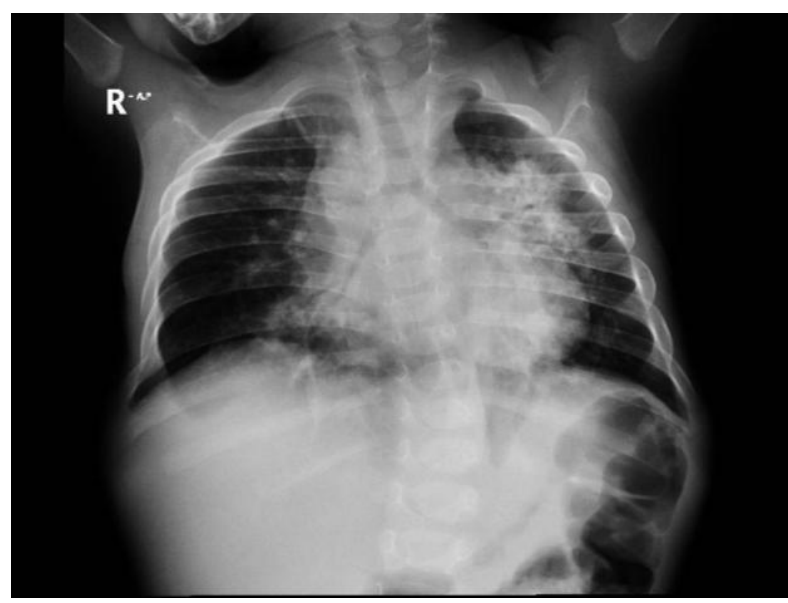

Fig-2: AP Chest radiograph showing severely hypoplastic lateral clavicles, slightly small and high scapulae, 11 pairs of ribs and mild levoscoliosis of the lower thoracic and upper lumbar spine.

There is also airspace disease in the left lung.

Radiograph of the thoracolumbar spine and pelvis showing mild levoscoliosis of the thoracolumar spine, mild hypoplasia of the iliac bones and the classic absent/delayed ossification of the pubic bone creating the appearance of a pseudo-widening of the symphysis pubis (figure 3).

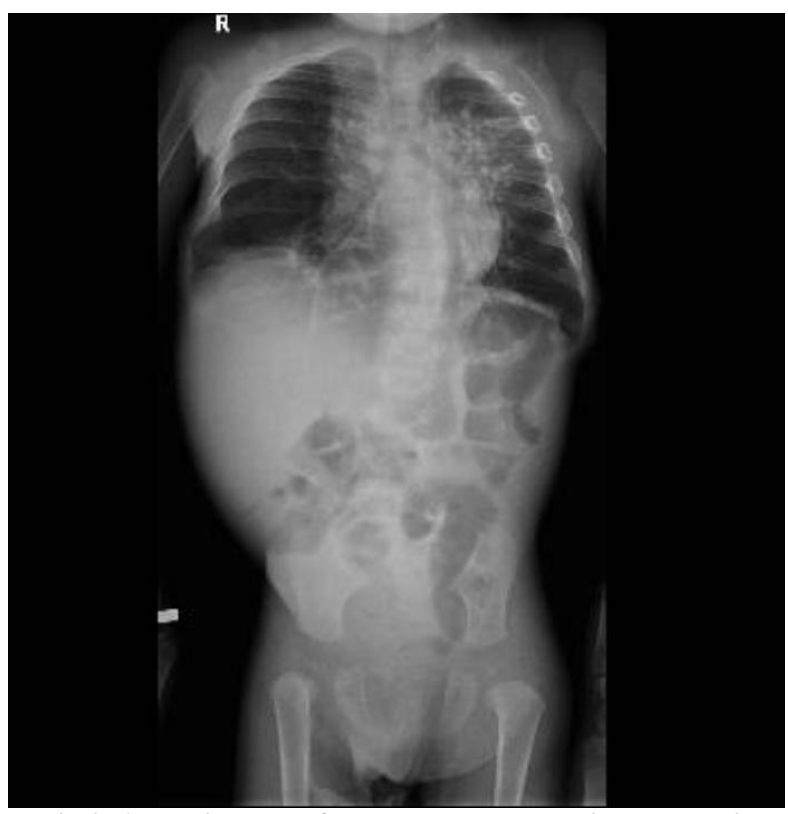

Fig-3: AP radiograph of the thoracolumbar spine and pelvis showing mild levoscoliosis of the thoracolumar spine, mild hypoplasia of the iliac bones and the classic absent/delayed ossification of the pubic bone creating the appearance of a pseudo-widening of the symphysis pubis 
Radiograph of both lower limbs showing mild enlargement of the femoral neck bilaterally especially in the left, very mild hypoplasia of the fibula bilaterally, in addition to the classic pseudo-widening of the symphysis pubis (figure 4).

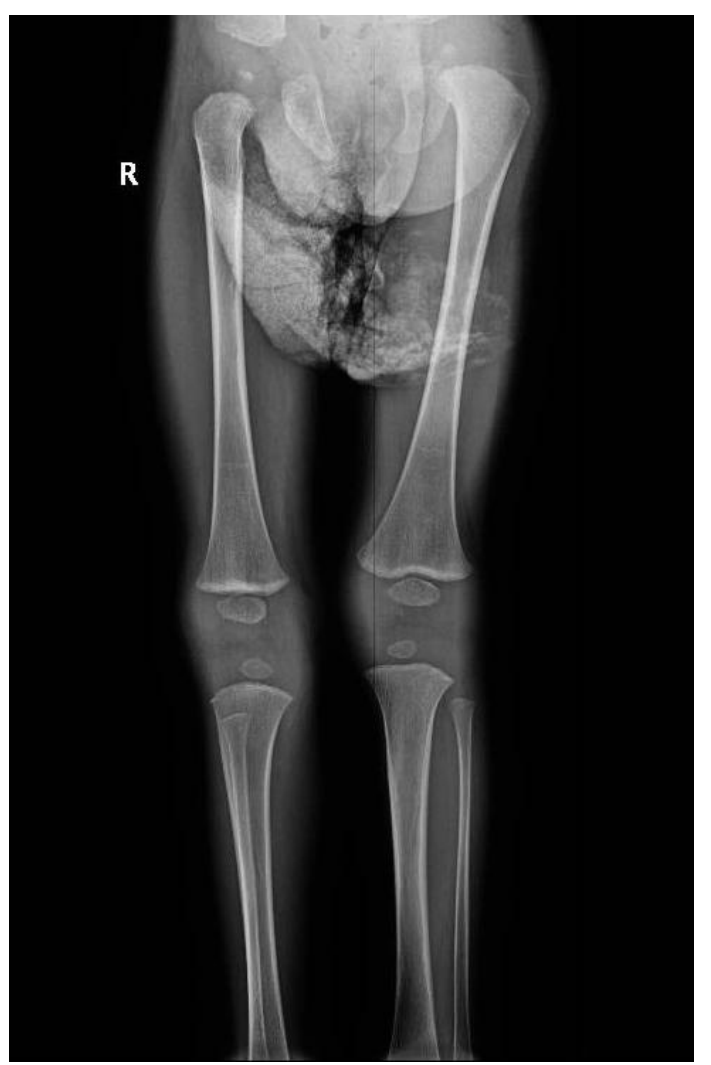

Fig-4: AP radiograph of both lower limbs showing mild enlargement of the femoral neck bilaterally especially in the left, very mild hypoplasia of the fibula bilaterally, in addition to the classic pseudo-widening of the symphysis pubis

\section{DisCUSSION}

CCD is inherited autosomal dominant trait disease in most cases; however in $20-40 \%$ of reported cases the disease occurs sporadically [1]. In the present case, there was no family history of CCD this main the family hadn't such mutation even on one allele as the parents were first degree consanguinity because it is a dominant trait which requires one allele to appear, so in this case the boy experienced sporadic mutation which result on CCD.

It was stated that CCD diagnosis is based on the clinical and radiological findings that present imaging of the thorax, cranium, pelvis and hands [5]. CCD is characterized by delayed closure of the cranial suture, short suture with brachycephalic skull, bossing partial and frontal bones, aplastic or hypoplastic clavicles, cone shaped thorax, multiple dental abnormalities such as retained primary teeth, delayed eruption of permenant teeth, supernumerary teeth and enamel hypoplasia $[9,10]$. The skeletal relationship of the jaws tend to be classified in class III position due to decreased vertical growth and the presence of hypoplastic maxilla [11]. Furthermore, other bony abnormalities can be occurring for CCD patients such as scoliosis [12]. On clinical examination, the boy of the current case was found to have supernumerary teeth and prominent forehead and on radiological examination by X-ray, he was found to have slightly prominent adenoid with patent postnasal air spaced, no clavicles were seen on both sides, whereas large anterior fontanel with open sutures were seen, hypoplastic maxilla, scoliotic deformity of lumbar spine, absence of carpal bones, accentuated bronchovascular marking noted in both hilar and perihilar region patchy air space shadowing seen in both perihilar region and upper zones and right middle lobe along right heart border. It was stated that the overall health of CCD patients is commonly good and the intellect isn't affected [5]. This was confirmed in the present case he has normal health with normal laboratory investigation.

\section{ACKNOWLEDGMENT}

We are greatly acknowledged to the patient family for their cooperation.

\section{CONCLUSion}

Our patient was suffering CCD due to sporadic mutation as his family has no history of such disease. We recommend doing a genetic predictive study for relatives of CCD patients because; they have a $50 \%$ chance to get inheritance of this mutation.

\section{REFERENCES}

1. McNamara CM, O'Riordan BC, Blake M, Sandy JR. Cleidocranial dysplasia: radiological appearances on dental panoramic radiography. Dentomaxillofac Radiol. 1999;28:89e97.

2. Alharbi AS, Ahmed SS. Cleidocranial Dysostosis: Report of Case from Saudi Population with New Radiographic Finding in Infraorbital Rim. American Journal of Medical Case Reports. 2018;6(8):149-51.

3. Mundlos S, Mulliken JB, Abramsom DL, Warman ML, Knoll JH, Olsen BR. Genetic mapping of cleidocranial dysplasia and evidence of a microdeletion in one family. Human molecular genetics. 1995 Jan 1;4(1):71-5.

4. Medina O, Muñoz N, Moneriz C. Cleidocranial dysplasia: a case report. Rev Chil Pediatr. 2017; 88(4): 517-523.

5. Buainain RA, Aruveetil YA, Alsineedi F, Alshammari AF, Ahmed HG. A 9-year-old Saudi Boy with Cleidocranial Dysplasia: A Case Report. American Journal of Medical Case Reports. 2017;5(11):270-3.

6. Otto F, Kanegane H, Mundlos S. Mutations in the RUNX2 gene in patients with cleidocranial dysplasia. Human Mutation. 2002; 19: 209-16.

7. Machuca-Tzili L, Monroy-Jaramillo N, Gonzálezdel Angel A, Kofman-Alfaro S. New mutations in 
the CBFA1 gene in two Mexican patients with cleidocranial dysplasia. Clin Genet. 2002; 61: 34953.

8. Yoshida T, Kanegane H, Osato M, Yanagida M, Miyawaki T, Ito Y, Shigesada K. Functional analysis of RUNX2 mutations in Japanese patients with cleidocranial dysplasia demonstrates novel genotype-phenotype correlations. Am J Hum Genet 2002; 71: 724-38.

9. Mundlos S. Cleidocranial dysplasia: clinical and molecular genetics. J Med Genet. 1999; 36: 177182.
10. Cavanaugh R, Croll RP. Enamel color modification by controlled hydrochloric acid-pumice abrasion. I technique and examples. Quintessence international (Berlin, Germany: 1985). 1986.

11. Richardson A and Deussen FF: Facial and dental anomalies in cleidocranial dysplasia: A study of 17 cases. Int J Paediatr Dent 4: 225- 231, 1994.

12. Cooper SC, Flaitz CM, Johnston DA, Lee B and Hecht JT: A natural history of cleidocranial dysplasia. Am J Med Genet. 104: 1-6, 2001. 